Volume 2

\title{
Tertiary Center Experience of Catheter-Directed Thrombolysis for Immediately Threatened Acute Lower Limb Ischemia of Native Vessels and Bypass Graft Thrombosis
}

Hani Hashim

M. Fuad Jan

Maharaj Singh

Suhail Allaqaband

Tanvir Bajwa

Anjan Gupta

Follow this and additional works at: https://aah.org/jpcrr

Part of the Cardiovascular Diseases Commons, and the Cardiovascular System Commons

\section{Recommended Citation}

Hashim H, Jan M, Singh M, Allaqaband S, Bajwa T, Gupta A. Tertiary Center Experience of CatheterDirected Thrombolysis for Immediately Threatened Acute Lower Limb Ischemia of Native Vessels and Bypass Graft Thrombosis. J Patient Cent Res Rev 2015;2:210-211. http://dx.doi.org/10.17294/

2330-0698.1235

Published quarterly by Midwest-based health system Advocate Aurora Health and indexed in PubMed Central, the Journal of Patient-Centered Research and Reviews (JPCRR) is an open access, peer-reviewed medical journal focused on disseminating scholarly works devoted to improving patient-centered care practices, health outcomes, and the patient experience. 
experiences, perceptions and preferences for how to learn TACT-associated skill sets to improve their competence as teachers.

Methods: A 7-question needs assessment survey was distributed to teaching faculty members in family medicine, internal medicine and ob/gyn in a health care system. Ranking, rating and free-response item formats were used to determine teachers' prioritization of care management and patient satisfaction metrics within medical education and their perceived skills and limitations in incorporating these factors into medical education. Data was analyzed using descriptive statistics and narrative comments using qualitative thematic analysis. This project was deemed "not human subjects research" by Aurora Health Care.

Results: A 78\% response rate was obtained (32/41). Respondents' top 3 teaching priorities were "Meeting specific clerkship objectives/residency milestones," "Impact on your time/teaching efficiency" and "Service quality priorities for the clinic." Respondents ranked learner's evaluation of teaching among their lowest priorities. $63 \%$ of respondents reported that they involve learners in improvement efforts (quality, safety, patient experience). Respondents identified a variety of strategies for involving learners in improvement efforts (medical students initiate patient callback, follow up on lab tests, check/address health maintenance items; residents identify a care management target), although time was consistently identified as a barrier to learner involvement.

Conclusion: Survey results confirmed that clinical teachers place value on integrating efforts to enhance clinical quality/ patient experience as they teach yet face challenges to TACT goal attainment. Findings will inform description of successful TACT strategies, assessment of their effectiveness and faculty development initiatives.

\section{Reducing Readmission Rates in Acute Pancreatitis Through Patient Education and Risk Assessment}

\section{Jordan T. Vulcano}

\section{Department of Internal Medicine, Aurora Sinai Medical Center}

Background: Early hospital readmissions are a direct burden on both our patients' well-being and health care system as a whole. Acute pancreatitis is a top offender, with countless 30-day readmissions. Studies have showed a consistently higher than average 30-day readmission rates in acute pancreatitis, around $19 \%$. This is significantly higher than the average all-cause readmission rate at Aurora Health Care hospitals. This quality improvement project aimed to reduce the rate of acute pancreatitis 30-day readmission rates at several Aurora hospitals through patient education and a readmission risk assessment tool.

Purpose: To clarify some of the risk factors associated with acute pancreatitis readmissions and reduce 30-day acute pancreatitis readmission rates through patient education and risk assessment to facilitate a safe discharge.
Methods: Project was conducted out of Aurora's Sinai, St. Luke's and West Allis Medical Centers with a total of 18 patients with acute pancreatitis admitted predominantly to the internal medicine teaching service between February 2014 and October 2014. Patients were seen within 1-2 days of admission and provided one-on-one education with a handout on acute pancreatitis. In addition, a 30-day pancreatitis readmission predictor (PRP) score was used to classify patient as low (5\%), moderate (17\%) or high $(68 \%)$ risk for readmission via Epic health record's "Dot Phrase." Subsequent readmissions, 14-day follow-up, total hospitalizations and emergency department visits were tracked through present. This was compared to readmission rates of a randomly selected control group of 18 patients admitted with acute pancreatitis.

Results: Patients had PRP scores ranging from 0 to 4 , with an average of 1 (rounded from 0.78 ). Of the 18 patients in the study group, only 2 were readmitted within 30 days for pancreatitis, or $11.1 \%$. The control group had 3 (16.7\%) readmissions within 30 days. Patients with alcohol-related pancreatitis were more likely to have a higher PRP (1.0) and readmission rate $(20 \%, 2 / 10)$.

Conclusion: A diagnosis of acute pancreatitis places the patient at a significantly higher than average risk of readmission. This project was able to reduce readmission rates from $16.7 \%$ to $11.1 \%$ by simple patient education and readmission risk assessment. Readmissions are detrimental to both the patient and health care system. This project serves as a starting point for reducing readmissions not only in acute pancreatitis patients but potentially other diagnosisspecific readmission initiatives.

\section{Tertiary Center Experience of Catheter-Directed Thrombolysis for Immediately Threatened Acute Lower Limb Ischemia of Native Vessels and Bypass Graft Thrombosis}

Hani Hashim, M. Fuad Jan, Maharaj Singh, Suhail Allaqaband, Tanvir Bajwa, Anjan Gupta

\section{Aurora Cardiovascular Services, Aurora Health Care; Aurora Research Institute, Aurora Health Care}

Background: Catheter-directed thrombolysis (CDT) is an effective therapy and a class I indication for patients with acute limb ischemia (ALI, Rutherford categories I and IIa) of less than 14 days duration, and class IIb indication for ALI (Rutherford category IIb) with symptoms more than 14 days duration. However, there is no consensus on the initial management option for ALI (Rutherford category IIb) with symptoms less than 14 days duration.

Purpose: To evaluate the safety, efficacy and outcome of CDT, with or without bailout Angiojet mechanical thrombectomy, in patients with immediately threatened acute lower extremity ischemia (Rutherford category IIb) as a minimally invasive alternative to emergent surgical revascularization.

Methods: We retrospectively reviewed data on 69 
consecutive patients (mean age $67 \pm 14.15$ years, $50.72 \%$ women) with ALI (Rutherford category IIb) who underwent CDT only (57.9\%) or CDT plus bailout Angiojet mechanical thrombectomy $(36.78 \%)$ at Aurora St. Luke's Medical Center from January 2004 to October 2014. Data were collected from electronic medical records, procedures reports, laboratory data and billing codes. Continuous variables were expressed as means \pm standard deviation and range; categorical variables were expressed as frequency count and percentage.

Results: Sites of target vessel for CDT were native vessel arterial thrombosis $(68.11 \%)$ and vascular bypass graft thrombosis $(27.5 \%)$. Reestablishment of blood flow and clinical success was achieved in $75.4 \%$ of patients, while limb salvage at 30 days was achieved in $87.1 \%$. Amputation at 30 days occurred in $12.9 \%$. Surgical embolectomy was required in $15.9 \%$, and lower extremity bypass surgery was required in $8.7 \%$. Time to lysis was $26.12 \pm 18.6$ hours. Bleeding complications that required blood transfusion occurred in $21 \%$ and hemorrhagic stroke in $1.44 \%$.

Conclusion: Catheter-directed thrombolysis for acute limb ischemia with symptoms less than 14 days (Rutherford category $\mathrm{Ilb}$ ) in native artery or bypass graft thrombosis has high immediate clinical success rate and very high limb salvage rate at 30 days. CDT is a reasonable minimally invasive alternative option to emergent surgical revascularization.

\section{PRACTC: Practice Readiness Academic Clinical Training Collaborative - Gap Analysis to Advance Clinical Training for Nurse Practitioner Students}

Jennifer Hartlaub, Mary Ann Muzi, M. Jamie Cairo, John R. Brill, James Weese, Kristin Rivera, Susan Hafemann, Ann M. Rohrer, Julia Schumacher, Terri L. Vandenhouten

Departments of Family Medicine and Academic Affairs, Aurora UW Medical Group; Departments of Oncology and Medical Education, Aurora Health Care

Background: Multiple factors have created a perfect storm of health care provider shortages in the United States. Advanced practice registered nurses (APRNs), long established as high-quality, cost-effective health care providers, are meeting health care needs across the nation in a variety of settings, and in Wisconsin will be needed to augment the primary care workforce. With $5.7 \%$ of its registered nurses credentialed as APRNs, Wisconsin lags behind the national average of $8.7 \%$. However, current capacity to educate this workforce is strained, requiring innovative data-driven clinical education models.

Purpose: To identify gaps in the current clinical educational framework for nurse practitioner (NP) students within the integrated health system.

Methods: Multiple data sources were used including NP core learning goals achievement, current continuum education/training experience models, health care system stakeholders' perspectives, and advanced practice provider hiring targets for 2015. NP-partnering universities' curricula and experiences of placing students within the integrated health system were reviewed. Analysis was conducted by an interprofessional team to identify gaps.

Results: Four gaps were identified: 1) structured learning and assessments focused on value-based care models (e.g. population, chronic disease) and tracking competencybased milestone achievement; 2) streamlined NP student placement system and onboarding through centralized onestop infrastructure; 3 ) interdisciplinary education to emulate theworkplacein which practice-ready graduates willbeplaced; and 4) number of preceptors with skills and knowledge regarding NP educational curriculum and competencies.

Conclusion: Systematic gap analysis will guide NP student placement and education at large Midwestern integrated health system. A structured clinical academic partnership with local university NP programs (PRACTC) that addresses preclinical preparedness, a structured student placement process, coordinated clinical experiences, preceptor development strategies and a diversity strategy provides a mechanism for accomplishing these goals.

\section{$\beta$-Thujaplicin: A Soil Antifungal}

\section{Dennis J. Baumgardner}

Department of Family Medicine, Aurora UW Medical Group; Center for Urban Population Health

Background: $\beta$-thujaplicin ( $\beta$-Th), also known as hinokitiol, naturally occurs in cedar mulch, is found in personal care products and has in vitro antitumor activities. It is antibacterial and antifungal, but has not been tested on soil. Scedosporium apiospermum (Sce) is an emerging "extremophile" fungal pathogen found in built outdoor environments.

Purpose: Pilot $\beta$-Th as "natural" soil antimicrobial or for isolation of extremophiles, and to explore $\beta$-Th resistance as selective advantage to Sce in mulched landscape.

Methods: A variety of outdoor and indoor environments were used for 2 sets of 24 paired soil samples. Soil/H20 slurry $(0.1 \mathrm{ml})$ was spread on Sabouraud dextrose agar with titrated $\beta$-Th levels of $0,25,250$ and $500 \mathrm{mg} / \mathrm{L}$ at $20^{\circ} \mathrm{C}$. Fungal and bacterial growth was semiquantitated with 4-point Likert scale. Wilcoxon signed rank test was used for comparison. A local soil Sce isolate was tested on each $\beta$-Th concentration. Results: There was no significant inhibition of total bacterial growth at $\beta$-Th $250 \mathrm{mg} / \mathrm{L}$ (mean 1.7/4) or $500 \mathrm{mg} / \mathrm{L}$ (mean 1.7) compared to plain Sabouraud dextrose agar (mean 1.6). Purple bacteria seemed to be selected for by $\beta$-Th. Fungal inhibition was essentially complete, similar, and significantly different from no $\beta$-Th (mean 3.4/4) at levels of 250 (mean 0.1 ) and $500 \mathrm{mg} / \mathrm{L}$ (mean 0.0). There was no significant fungal inhibition at $25 \mathrm{mg} / \mathrm{L}$ (mean 3.2 , second set samples). Similarly, Sce was completely inhibited at 250 and $500 \mathrm{mg} / \mathrm{L}$, but not inhibited at $25 \mathrm{mg} / \mathrm{L}$.

Conclusion: In vitro, $\beta$-thujaplicin profoundly, but 